\title{
Transport in graphene superimposed by a moving electrical superlattice potential
}

\author{
Jürgen Dietel ${ }^{1}$ and Hagen Kleinert ${ }^{1,2}$ \\ ${ }^{1}$ Institut für Theoretische Physik, Freie Universität Berlin, Arnimallee 14, D-14195 Berlin, Germany \\ ${ }^{2}$ ICRANeT, Piazzale della Repubblica 1, 10-65122, Pescara, Italy
}

(Received 12 July 2012; published 27 September 2012)

\begin{abstract}
We calculate dc conductivities of ballistic graphene undulated by an overlying moving unidirectional electrical superlattice (SL) potential whose SL velocity is smaller than the electron velocity. We obtain no dependence of the conductivity on the velocity along the direction of the superlattice wave vector. In the orthogonal direction however, the dependence is strong on the velocity especially at voltages where a new Dirac point emerges for zero velocity. It is shown that the infinite graphene system can serve as an ideal motion detector at potentials where the first new Dirac point emerges. There the conductivity is zero at vanishing SL velocities and jumps to infinity when the SL starts moving. For finite systems at voltages where the number of new Dirac points is of the order of the ratio of the electron velocity by the SL velocity, the modifications to the conductivity of a moving $\mathrm{SL}$ is at least of similar magnitude as the conductivity of the stagnant SL.
\end{abstract}

DOI: 10.1103/PhysRevB.86.115450

PACS number(s): 72.80.Vp, 73.21.Cd, 73.22.Pr

\section{INTRODUCTION}

The electrical conductivities in suspended graphene samples show high mobilities where ballistic transport is seen for samples up to the micron length. ${ }^{1-3}$ Due to the quasirelativistic behavior of its electrons, graphene has a density of states proportional to the electronic energy, which is zero at the neutrality point. As a consequence, this leads in ballistic graphene to the phenomenon that the conductivity shows a universal finite behavior ${ }^{4}$ whose precise value is still under debate. ${ }^{5}$ It seems now that the universal conductivity in a wide range of graphene samples with highly doped leads has the value $\tilde{\sigma}=4 e^{2} / \pi h{ }^{6,7}$ whereas in a system with vanishing small doped leads, it is $\tilde{\sigma}=e^{2} \pi / 2 h{ }^{8}$ Numerically, these two values are quite close to each other. A small perturbation of the chemical potential of the graphene sample may be caused by applying an external gate voltage; this conductivity can change drastically, due to the now finite density of states at the Fermi energy. For an infinite large ballistic system, it becomes even infinite. Such an extreme sensitivity of the neutral graphene system on the environmental parameters makes it attractive as a building block for nanodetectors. It was experimentally shown that graphene is a good chemical sensor which is able to detect the dc-response changes due to the adsorption of even single gas molecules on its surface. ${ }^{9}$ This high sensitivity is mainly due to the intrinsic low-noise properties of graphene. A more general review of possible graphene sensors can be found in Ref. 10.

Here we consider a ballistic graphene sample with an overlying slowly moving unidirectional electrical superlattice (SL). We calculate the longitudinal conductivities along and orthogonal to the SL wave vector as a response of a small external dc field. This system is considered as a possible model for a graphene-based nanomechanical motion detector.

In the direction orthogonal to the wave vector of the SL we obtain, especially at SL voltages where new Dirac points emerge in the nonmoving SL, a high sensitivity of the conductivity values on the SL motion. In the parallel direction our approximation produces no dependence on the SL velocity.

Graphene under the effect of a moving SL can be realized, for example, by placing periodically patterned gate electrodes on either a moving underlying substrate or on a rested substrate where now the individual gate electrodes are activated appropriately with time such that an effective moving SL is simulated. More directly, the experimental realization could also be carried out by using the coupling of the graphene sheet to the electrical field of a surface acoustic wave on a piezoelectric substrate ${ }^{11}$ or to a charged moving membrane with ripples.

It was recently shown explicitly for graphene that new Dirac points in the energy spectrum can be opened by imposing a nonmoving SL on the graphene lattice. ${ }^{12-14}$ This leads to unusual conductivity properties in such systems. ${ }^{15-20}$ These new Dirac points are accompanied with new energy valleys. Due to the technical complications in handling transport in a moving SL we will first consider the transport contributions of the inner valleys near the $\mathbf{K}$ and $\mathbf{K}^{\prime}$ points in Sec. III, then those of the outer valleys in Sec. IV. Note, that such a separation is not useful for the nonmoving SL, as will be seen in Sec. IV. We start in Sec. II by first reconsidering the lowestband eigenvalues and eigenfunctions for the nonmoving SL.

We discuss here the most simple representation of a SL, a symmetric two-step Kronig-Penney potential with a superlattice potential $V(x)=V \chi(x)$, where $\chi(x)=\operatorname{sg}[\sin (2 \pi x / d)]$ (cf. Fig. 1). The function $\operatorname{sg}[x]$ is the sign of $x$, and $d$ is the wavelength of the SL. In the continuum approximation, the graphene Hamiltonian under consideration near the Dirac point $\mathbf{K}$ is given by $H_{v_{s}}=\hbar v_{F}\left(\sigma_{x} \partial_{x} / i+\sigma_{y} \partial_{y} / i\right)+V(x+$ $\left.v_{s} t\right) .{ }^{4}$ Here $v_{F}$ is the Fermi velocity and $\sigma_{x, y}$ are the Pauli matrices, while $v_{s}$ is the velocity of the moving SL. Before starting, we mention here that we kept track of the most important in-line formulas in this paper in Table I. This should enable the reader to better capture the structure of the paper. Furthermore, we give a short guideline for reproducing the formulas used in this paper in Appendix B.

\section{LOWEST-BAND EIGENVALUES AND EIGENFUNCTIONS}

In the following, we solve the eigenvalue equation $H_{v_{s}} \mathbf{u}^{v_{s}}\left(\mathbf{r}^{\prime}\right)=\epsilon \mathbf{u}^{v_{s}}\left(\mathbf{r}^{\prime}\right)$ for a nonmoving SL $\left(v_{s}=0\right)$ by using 


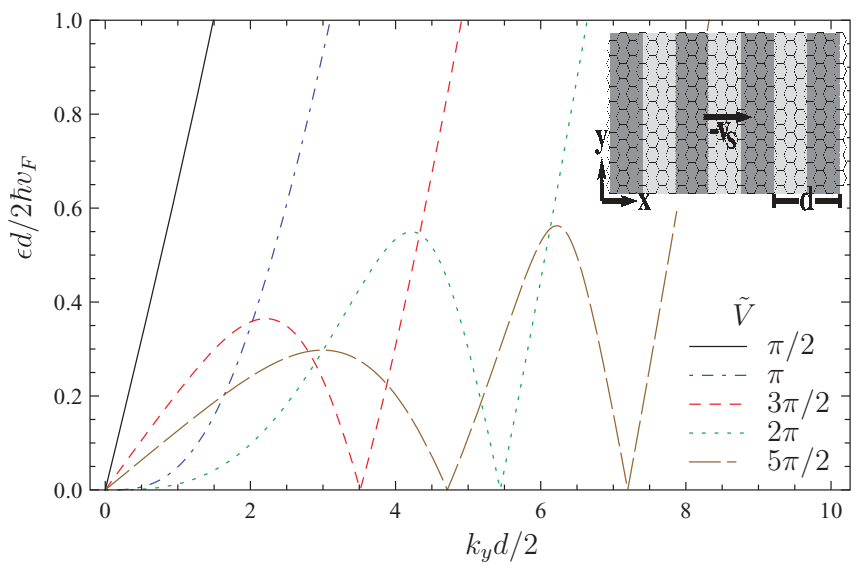

FIG. 1. (Color online) Lowest Bloch band energy spectrum for $k_{x}=0$ as a function of dimensionless momenta $k_{y}$ for various SL potential strengths $\tilde{V}$ (the full lowest-band energy spectrum can be obtained by using its mirror symmetry with respect to the $x, y$ axis). Here we used the transfer-matrix method (Refs. 21 and 16). Inset shows a graphene layer with an overlying moving SL in the $x$ direction.

the transfer-matrix method. ${ }^{16,21}$ For the energy dispersion in the lowest band we restrict ourselves to the lowest-lying oscillatory regime $\left|\epsilon_{s}\right| d / \hbar v_{F} \ll \tilde{V}, \tilde{\alpha}_{0}$ and obtain ${ }^{22}$

$$
\epsilon_{s}=s \hbar v_{F} \tilde{\alpha}_{0}^{2} \sqrt{k_{x}^{2}+|\Gamma|^{2} k_{y}^{2}},
$$

with

$$
\alpha_{\epsilon_{s}}(x)=\left(\left\{\left[\epsilon_{s}-V(x)\right] / \hbar v_{F}\right\}^{2}-k_{y}^{2}\right)^{1 / 2} d / 2 .
$$

Here $\Gamma=\sin \left[\alpha_{0}\right] e^{i \alpha_{0}} / \alpha_{0}$ and $\hat{\alpha}_{0}=\alpha_{0} / \tilde{V}$, where $\tilde{V}=$ $V d / 2 \hbar v_{F}$. The Bloch momentum in the $x$ direction is restricted to $-\pi / d \leqslant k_{x} \leqslant \pi / d$. The parameter $s=1$ denotes the conduction band and $s=-1$ the valence band.

The corresponding lowest-band eigenfunctions are $\mathbf{u}^{v_{s}}(x, y)=e^{i k_{y} y} \mathbf{u}^{v_{s}}(x)$, in the fundamental zone $0 \leqslant x \leqslant d$ and for $v_{s}=0$ reduces to

$$
\mathbf{u}_{s}^{0}(x)=\Lambda(x) \mathbf{u}_{s}^{0}(0)
$$

with

$$
\Lambda(x)=\lambda_{0}(x) \Theta\left(\frac{d}{2}-x\right)+\lambda_{d / 2}(x) \lambda_{0}\left(\frac{d}{2}\right) \Theta\left(x-\frac{d}{2}\right),
$$

TABLE I. Overview of the most important in-line formulas in this paper.

\begin{tabular}{lc}
\hline \hline$\chi(x)=\operatorname{sg}[\sin (2 \pi x / d)]$ & $\xi(x)=\int_{0}^{x} d x^{\prime} \chi\left(x^{\prime}\right)$ \\
$\Gamma=\sin \left[\alpha_{0}\right] e^{i \alpha_{0}} / \alpha_{0}$ & $\hat{\alpha}_{0}=\alpha_{0} / \tilde{V}$ \\
$\tilde{V}=V d / 2 \hbar v_{F}$ & $\tilde{k}_{y}=\hbar v_{F} k_{y} / V$ \\
\hline Inner valleys & Outer valleys \\
\hline$\hat{\alpha}_{0} \approx \frac{\pi n}{\tilde{V}}$ & $\hat{\alpha}_{0}=\frac{\pi n}{\tilde{V}}$ \\
$k_{y}^{n} d \approx 2[2 \tilde{V}(\tilde{V}-\pi n)]^{1 / 2}, k_{y}^{0}=0$ & $k_{y}^{n} d=2\left[\tilde{V}^{2}-(\pi n)^{2}\right]^{1 / 2}$ \\
$\Gamma_{n} \approx 2\left(1-\frac{\pi n}{\tilde{V}}\right), \Gamma_{0}=\frac{\sin (\tilde{V})}{\tilde{V}}$ & $\Gamma_{n}=1-\left(\frac{\pi n}{\tilde{V}}\right)^{2}$ \\
\hline \hline
\end{tabular}

where

$$
\lambda_{x_{0}}(x)=\cos \left[\frac{\alpha_{\epsilon_{s}}(x) 2\left(x-x_{0}\right)}{d}\right] \mathbf{E}+\frac{\sin \left[\frac{\alpha_{\epsilon_{s}}(x) 2\left(x-x_{0}\right)}{d}\right]}{\alpha_{\epsilon_{s}}(x)} \mathbf{M}
$$

Here $\mathbf{E}$ is the unit matrix and

$$
\mathbf{M}=k_{y} \sigma_{3}+i\left[\epsilon_{s}-V(x)\right] \sigma_{2} / \hbar v_{F} .
$$

$\mathbf{u}_{s}^{0}(0)$ is given in the oscillatory region $\left|\epsilon_{s}\right| d / \hbar v_{F} \ll \tilde{V}, \hat{\alpha}_{0}$ by

$$
\mathbf{u}_{s}^{0}(0) \approx \frac{1}{N_{u}}\left(\begin{array}{c}
\frac{\cos \left(\alpha_{0}\right) \sin \left(\alpha_{0}\right)}{\alpha_{0}} k_{y} d+i k_{x} d \\
i \frac{1}{\hat{\alpha}_{0}^{2}} \frac{\epsilon_{s} d}{\hbar v_{F}}+i \frac{\sin ^{2}\left(\alpha_{0}\right)}{\alpha_{0}^{2}} \tilde{V} k_{y} d
\end{array}\right),
$$

where $N_{u}$ in (7) denotes a normalization factor. From (1) we obtain an oscillatory behavior of the lowest-band eigenvalues as a function of $k_{y}$ (cf. Fig. 1). New Dirac points emerge at $\mathbf{k}=0$ for $\tilde{V} \in \mathbb{N} \pi$. These are shifted along the $y$ axis in $\mathbf{k}$ space for increasing $\tilde{V}$. Note that the lowest-band energy values beyond the oscillatory regime with momenta $k_{y}^{2} \gg\left(V / \hbar v_{F}\right)^{2}$ scale like $\left|\epsilon_{s}\right| \sim \hbar v_{F}\left|k_{y}\right| .^{16,21}$

In the following, we discuss the transport contributions of electrons in the inner-energy valleys where $k_{y} \ll V / \hbar v_{F}$ and the outer valleys where $\hat{\alpha}_{0} \ll 1$ separately. Such a separation is possible for $\mathrm{dc}$ transport since as we will see in the following, the dc electric field couples only electron states in the conduction and valence bands having the same Bloch momentum. Note that the Bloch momentum is conserved for a moving SL. The resulting time-dependent state then performs a similar movement as is known under the Zitterbewegung in relativistic physics. ${ }^{8}$ Taking into account all electrons in the valence band we obtain for large times an effective dc current.

\section{INNER-VALLEY TRANSPORT CONTRIBUTIONS}

In the inner-valley regime $k_{y} \ll V / \hbar v_{F}$, the lowest-band eigenfunctions $\mathbf{u}_{s}^{0}(x)[(3)-(7)]$ for the nonmoving system above are given by

$$
\begin{aligned}
\mathbf{u}_{s}^{0}(x, t)= & \frac{1}{N_{u}}\left[\left(\begin{array}{l}
1 \\
1
\end{array}\right)\left(\frac{-i k_{x}}{k_{y} \Gamma^{*}}-\frac{i \epsilon_{s}}{\hbar v_{F} k_{y} \Gamma^{*}}\right) \phi_{+}(x)\right. \\
& \left.+\left(\begin{array}{c}
-1 \\
1
\end{array}\right) \phi_{-}(x)\right],
\end{aligned}
$$

where $\tilde{N}_{u}$ in (8) denotes a normalization factor. $\Gamma^{*}$ is the complex conjugate of $\Gamma$. The phase factor $\phi_{ \pm}(x)$ is given by

$$
\phi_{ \pm}(x, t)=\exp \left[i S_{ \pm}^{v_{s}}(x, t) / \hbar\right]
$$

with

$$
S_{ \pm}^{0}(x, t)=\mp i \hbar \int_{0}^{x} d x^{\prime} \operatorname{sg}\left[V\left(x^{\prime}\right)\right] \alpha_{\epsilon_{s}}\left(x^{\prime}\right) /(d / 2)-i \epsilon_{s} t
$$

for $v_{s}=0$ where we extended (8) by the last term in (10) chosen such that $\mathbf{u}_{s}^{0}$ solves simultaneously the corresponding time-dependent Schrödinger equation (TSE). From (8) we deduce the remarkable observation that the inner-valley electrons do not backscatter at the potential steps. This phenomenon is well known for ordinary Dirac fermions as Klein paradox.

In the following, we use the inner-valley approximation

$$
\alpha_{0}(x) \approx \tilde{V}\left(1-\tilde{k}_{y}^{2} / 2\right)
$$


with $\tilde{k}_{y}=\hbar v_{F} k_{y} / V$ in (1) and (8), which is a good approximation of the overall oscillatory behavior of the energy dispersion in Fig. 1. Similar approximations will also be used when solving the TSE for $v_{s} \neq 0$ below. Finally we note that the missing of the $k_{x}, k_{y}$ dependence in the vector part $(\mp 1,1)^{T}$ of both spinor components in (8) is due to the inner-valley restriction $k_{y}^{2} \ll\left(V / \hbar v_{F}\right)^{2}$.

We obtain from (1) that an entire set of $2[\tilde{V} / \pi]+1$ Dirac points exists near $\mathbf{K}$ where $[x]$ is the lowest integer number smaller than $x$. By using the inner-valley approximation (11), these new Dirac points are located at $k_{y}^{n} d \approx 2[2 \tilde{V}(\tilde{V}-\pi n)]^{1 / 2}$ with $n=1, \ldots,[\tilde{V} / \pi]$ and $k_{y}^{0}=$ 0 (restricting ourselves to positive $k_{y}$ ). The linearized energy spectrum around these Dirac points is given by $\epsilon_{s}^{n}=$ $s \hbar v_{F}\left[k_{x}^{2}+\Gamma_{n}^{2}\left(k_{y}-k_{y}^{n}\right)^{2}\right]^{1 / 2}$ where the effective $y$-velocity coefficient is given by $\Gamma_{n} \approx 2(1-n \pi / \tilde{V})$ for $n=1, \ldots,[\tilde{V} / \pi]$ and $\Gamma_{0}=\sin (\tilde{V}) / \tilde{V}$ for the central valley. The magnitude $\hat{\alpha}_{0}$ for $k_{y}=k_{y}^{n}$ is given by $\pi n / \tilde{V}$. Below, we shall also need the $k_{y}$-momentum spacings between the right- and left-energy crest and the Dirac point. The spacing for the right crest is given by $\Delta k_{y}^{n, R} d \approx \pi[\tilde{V} / 2(\tilde{V}-\pi n)]^{1 / 2}$ for $n=$ $1, \ldots,[\tilde{V} / \pi]$ and $\Delta k_{y}^{n, L}=\Delta k_{y}^{n, R}$ for the left crest positions where $n=1, \ldots,[\tilde{V} / \pi]-1$. For the central crest distance we obtain $\Delta k_{y}^{0, R} d=\Delta k_{y}^{0, L} d \approx\left(k_{y}^{[\tilde{V} / \pi]} d\right)^{3} /\left[\left(k_{y}^{[\tilde{V} / \pi]} d\right)^{2}+4 \Gamma_{0} \tilde{V}^{2}\right]$ and $\Delta k_{y}^{[\tilde{V} / \pi], L}=k_{y}^{[\tilde{V} / \pi]}-\Delta k_{y}^{0, L}$. Finally we note that the inner-valley formula with $\tilde{k}_{y}^{2} \ll 1$ considered in this section is valid for the valleys $1-\pi n / \tilde{V} \ll 1$ with $n \neq 0$ and also the central valley $n=0$.

In the following we solve the TSE $i \hbar \partial_{t} \mathbf{u}_{s}^{v_{s}}(x, t)=$ $H_{v_{s}} \mathbf{u}_{s}^{v_{s}}(x, t)$ with the initial condition $\mathbf{u}_{s}^{v_{s}}(x, 0)=\mathbf{u}_{s}^{0}(x)$ for $t=$ 0 in the oscillatory regime by using the above approximations. Note that by using the characteristic method we can solve the TSE without approximation for $k_{y}=0$. This leads again to (8) where now $S_{ \pm}^{v_{s}}$ is $v_{s}$ dependent. Instead of doing this explicitly, we can generalize this procedure to any nonzero $k_{y}^{2} \ll\left(V / \hbar v_{F}\right)^{2}$ by the Hamilton-Jacobi ansatz

$$
-\frac{\partial S_{ \pm}^{v_{s}}}{\partial t}=-\hbar v_{F} \operatorname{sg}\left[V\left(x+v_{s} t\right)\right] \sqrt{\left(\partial_{x} S_{ \pm}^{v_{s}}\right)^{2}+k_{y}^{2}}+V\left(x+v_{s} t\right)
$$

with the boundary condition that $S_{ \pm}^{v_{s}}(x, 0)=S_{ \pm}^{0}(x, 0)$. Due to the local uniformity of $V\left(x+v_{s} t\right)$ in position and time we obtain local uniform solutions of (12). That this approach leads to a TSE solution in the oscillatory regime is due to the fact that the general solution can be written as

$$
\mathbf{u}_{s}^{v_{s}}(x, t) \approx \sum_{s, k_{x}} a_{s, k_{x}}\left(\begin{array}{c}
s \frac{k_{x}}{\left|k_{x}\right|} \\
1
\end{array}\right) e^{-(i / \hbar) t\left[\hbar v_{F} s \sqrt{k_{x}^{2}+k_{y}^{2}}+V\left(x+v_{s} t\right)\right]} e^{i k_{x} x}
$$

in the inner-valley regime $k_{y}^{2} \ll k_{x}^{2}$. The complex variables $a_{s, k_{x}}$ are local uniform functions in the $(x, t)$ plane. We will show below that $a_{s, k_{x}}$ is nonzero for only two special $k_{x}$ values which moreover fulfill the inner-valley regime condition $k_{y}^{2} \ll k_{x}^{2}$.

We now solve (12) by using a generalized characteristic method for the Hamilton-Jacobi equation that is well known in the semiclassical approach to quantum mechanics. ${ }^{23}$ This is based on the one-particle mechanical trajectory of a relativistic particle and antiparticle in a step potential. The calculation is outlined in Appendix A.

After some manipulation we obtain the result

$$
S_{ \pm}^{v_{s}}(x, t) \approx S_{ \pm}^{v_{s}, x}+S_{ \pm}^{v_{s}, t}
$$

with

$$
\begin{aligned}
\frac{S_{ \pm}^{v_{s}, t}}{\hbar}= & -t \frac{\epsilon_{s}}{\hbar}, \\
\frac{S_{ \pm}^{v_{s}, x}}{\hbar}= & \pm\left[A_{ \pm} \xi\left(x^{*}+v_{s}^{*} t\right)+B_{ \pm} \xi\left(x^{*} \mp v_{F}^{*} t\right)\right. \\
& \left.+C_{ \pm} t \chi\left(x^{*} \mp v_{F}^{*} t\right)\right] .
\end{aligned}
$$

Further we have $\xi(x)=\int_{0}^{x} d x^{\prime} \chi\left(x^{\prime}\right), x^{*}=x-\left(v_{s}^{*}-v_{s}\right) t$, and

$$
\begin{aligned}
A_{ \pm} & =\mp \frac{V}{\hbar\left(v_{s} \pm v_{F}\right)}\left[1-\tilde{k}_{y}^{2} \frac{v_{s} \pm \frac{1}{2} v_{F}}{v_{s} \pm v_{F}}+\tilde{k}_{y}^{2} Z_{ \pm}^{2}\right], \\
B_{ \pm} & =-\frac{V}{\hbar\left(v_{s} \pm v_{F}\right)}\left[\frac{v_{s}}{v_{F}}-\frac{\tilde{k}_{y}^{2} v_{s}}{2 v_{F}} \frac{v_{s}}{v_{s} \pm v_{F}} \mp \tilde{k}_{y}^{2} Z_{ \pm}^{2}\right], \\
C_{ \pm} & = \pm \frac{V}{\hbar} \tilde{k}_{y}^{2} Z_{ \pm}^{1}, \quad v_{F}^{*}=v_{F}\left[1-\frac{\tilde{k}_{y}^{2}}{2} \frac{\left(v_{s}^{2}+v_{F}^{2}\right)^{2}}{\left(v_{s}^{2}-v_{F}^{2}\right)^{2}}\right], \\
v_{s}^{*} & =v_{s}\left[1-\tilde{k}_{y}^{2} \frac{v_{F}^{2}\left(v_{s}^{2}+v_{F}^{2}\right)}{\left(v_{s}^{2}-v_{F}^{2}\right)^{2}}\right],
\end{aligned}
$$

where $Z_{ \pm}^{1}=v_{s}^{3}\left(v_{s} \pm v_{F}\right) /\left(v_{s}^{2}-v_{F}^{2}\right)^{2}$ and $Z_{ \pm}^{2}=v_{s}^{2} /\left(v_{s}^{2}-\right.$ $\left.v_{F}^{2}\right)$. Here we restrict the solution of (12) to small velocities $v_{s} \lesssim v_{F}$.

Next we calculate the dc response in the moving SL system. This is done in the gauge $\mathbf{A}=-c \mathbf{E}\left(t-t_{0}\right) \Theta\left(t-t_{0}\right)$ assuming $t_{0} \leqslant 0$, in general. Since $\tilde{\sigma}_{i i}(t)$ does not depend on $t_{0}$ for $t \gg 0$ we set immediately $t_{0}=0$. The total Hamiltonian in the continuum approximation is then given by $H_{A}=H_{v_{s}}+$ $\hbar v_{F}(e / c)\left(\sigma_{x} A_{x}+\sigma_{y} A_{y}\right)$. The corresponding TSE solution which we expand to first order in $\mathbf{A}$ and assume it to satisfy the initial condition $\mathbf{u}_{A}(t=0)=\mathbf{u}_{s}^{0}$ is denoted by $\mathbf{u}_{A}$. From this solution we obtain the conductivity in the $i$ th direction by $\tilde{\sigma}_{i i}=\lim _{E \rightarrow 0} e v_{F}\left(\left\langle\mathbf{u}_{A}(t) \sigma_{i} \mathbf{u}_{A}(t)\right\rangle / E\right)$ where $\mathbf{A}=-c E \mathbf{e}_{i} t$. Here $\mathbf{e}_{i}$ is the unit vector in the $i$ th direction. The conductivity in the $i$ th direction in the lowest energy level approximation valid for $t \rightarrow \infty$ and $v_{s} \lesssim v_{F}, V d / \hbar$ is then given by ${ }^{8}$

$$
\tilde{\sigma}_{i i}(t)=\frac{-4 e v_{F}}{(2 \pi)^{2}} \int_{\mathrm{BZ}} d^{2} k \operatorname{Re}\left[\left\langle\mathbf{u}_{-1}^{v_{s}}(t)\left|\sigma_{i}\right| \mathbf{u}_{+1}^{v_{s}}(t)\right) \xi_{+}(t)\right]
$$

with

$$
\begin{aligned}
\xi_{+}(t) & =i \frac{e v_{F}}{\hbar} \int_{0}^{t} d t^{\prime} t^{\prime} \mathcal{T}^{v_{s}}\left(t^{\prime}\right) \\
& =i \frac{e v_{F}}{\hbar}\left(t \int_{t^{\prime \prime}=-\infty}^{t} d t^{\prime \prime}-\int_{t^{\prime}=0}^{t} d t^{\prime} \int_{t^{\prime \prime}=-\infty}^{t^{\prime}} d t^{\prime \prime}\right) \mathcal{T}^{v_{s}}\left(t^{\prime \prime}\right)
\end{aligned}
$$

and the transition matrix element $\mathcal{T}^{v_{s}}(t)=\left\langle\mathbf{u}_{1}^{v_{s}}(t)\left|\sigma_{i}\right| \mathbf{u}_{-1}^{v_{s}}(t)\right\rangle$. By inserting (18) in (17) the term proportional to $t$ cancels in an improved tight-binding approximation since it can be written as $t \int_{\mathrm{BZ}} d^{2} k \partial_{k_{i}}\left\langle\mathbf{u}_{-1}^{v_{s}}(t)\left|J_{i}\right| \mathbf{u}_{-1}^{v_{s}}(t)\right\rangle=0$, where $J_{i}$ is the 
tight-binding current operator for $\mathbf{A}=0 .{ }^{8}$ Here we used the fact that the exact tight-binding wave functions are smooth at the Brillouin zone boundary. Summing the Fourier series $\sum_{\omega_{n}} \hat{\mathcal{T}}_{r}^{v_{s}}\left(\omega_{n}\right) e^{i \omega_{n} t} \equiv e^{-i t \Delta \epsilon / \hbar} \mathcal{T}^{v_{s}}(t)$ where $\Delta \epsilon=\epsilon_{1}-\epsilon_{-1}$, we obtain for large times

$$
\begin{aligned}
\xi_{+}(t)= & i \frac{e v_{F}}{\hbar} \sum_{\omega_{n}}\left\{\frac{\hat{\mathcal{T}}_{r}^{v_{s}}\left(\omega_{n}\right)}{\left(\omega_{n}+\Delta \epsilon / \hbar-i \delta\right)^{2}}\left(e^{i\left[(\Delta \epsilon / \hbar)+\omega_{n}\right] t}-1\right)\right. \\
& \left.-i t \frac{\left[\hat{\mathcal{T}}_{r}^{v_{s}}\left(\omega_{n}\right)-\hat{\mathcal{T}}_{r}^{0}\left(\omega_{n}\right)\right]}{\omega_{n}+\Delta \epsilon / \hbar-i \delta}\right\}
\end{aligned}
$$

Here $\delta$ is an infinitesimal positive number.
In the following we calculate the contribution of every energy valley to the momentum integral in (17) separately, i.e.,

$$
\tilde{\sigma}_{i i}(t)=\sum_{n=0, \ldots,[\tilde{V} / \pi]} \tilde{\sigma}_{i i}^{n}(t)\left(2-\delta_{n, 0}\right)
$$

For large times one can restrict the $k_{y}$ integrals of Eq. (17) to the neighborhood of the valley center $k_{y}^{n}$ setting immediately $k_{y} \approx k_{y}^{n}$ in $\hat{\mathcal{T}}_{r}^{v_{s}}$. This leads then with (8), (17), and (19) to the following momentum integrals during the calculation of $\tilde{\sigma}_{i i}^{n}$ :

$\int_{n \text {th valley }} d^{2} k \frac{\left(2 \hbar v_{F} k_{x}\right)^{2}}{(\Delta \epsilon)^{2}} e^{-(i / \hbar) \Delta \epsilon t} \xi_{+}(t)=\frac{i e}{2 \hbar} \frac{1}{\Gamma_{n}}\left\{\int_{-\infty}^{+\infty} d k_{x} \int_{-\Delta k_{y}^{n, L} \Gamma_{n}}^{\Delta k_{y}^{n, R} \Gamma_{n}} d k_{y} \frac{k_{x}^{2}}{k^{3}} F^{v_{s}}(k)-\int_{0}^{2 \pi} d \vartheta k_{\vartheta} \sin ^{2}(\vartheta)\left[F^{v_{s}}\left(k_{\vartheta}\right)-F^{0}\left(k_{\vartheta}\right)\right]\right\}$

with

$$
F^{v_{s}}(k)=\frac{\hat{\mathcal{T}}_{r}^{v_{s}}\left(\omega_{n}\right) e^{i \omega_{n} t}}{\omega_{n}+2 v_{F} k-i \delta}\left(1-e^{-i\left(2 v_{F} k+\omega_{n}\right) t}\right)
$$

and $k_{\vartheta}=\left[\tan ^{2}(\vartheta)+1\right]^{1 / 2} \Gamma_{n}\left\{\Delta k_{y}^{n, R} \Theta[\cos (\vartheta)]+\Delta k_{y}^{n, L} \Theta[-\cos (\vartheta)]\right\}$ where $\Theta(x)$ is the Heaviside function. The right-hand side of Eq. (21) was calculated by the help of a partial integration.

In the calculation of $\tilde{\sigma}_{i i}$ via (17), the quantities

$$
\mathcal{P} \equiv \frac{1}{d} \int_{0}^{d} d x \exp \left[i\left(S_{+}^{v_{s}, x}-S_{-}^{v_{s}, x}\right)\right], \quad \mathcal{C}_{m} \equiv \sum_{\omega_{n} \approx 2 m \pi v_{F}^{*} / d}|\hat{\mathcal{P}}|\left(\omega_{n}\right)\left(2-\delta_{n, 0}\right) e^{i \omega_{n} t}
$$

are relevant where $|\hat{\mathcal{P}}|\left(\omega_{n}\right)$ are the Fourier components of $|\mathcal{P}|(t)$. More precisely, $\mathcal{C}_{m}$ with $m>0$ are the positive components for frequencies $2 \pi(m-1 / 2) v_{F}^{*} / d \leqslant \omega_{n} \leqslant 2 \pi(m+1 / 2) v_{F}^{*} / d$ under the restriction that $\omega_{n} \geqslant 0$ for $m=0$. A straightforward calculation leads with (15) for $v_{s} \lesssim v_{F}$ to

$$
\begin{aligned}
& \operatorname{Re}\left[\mathcal{C}_{m}\right]=\sum_{\sigma \in\{ \pm\}} \cos \left(C_{-} t\right) B_{-} X(m, m, m, \sigma)-\sin \left(C_{-} t\right)\left[\sigma \frac{2 \pi m}{d}+\dot{\xi}\left(2 v_{s}^{*} t\right) B_{+}\right] X(m, m, m+1, \sigma)+\operatorname{Ex} \\
& \operatorname{Im}\left[\mathcal{C}_{m}\right]=\sum_{\sigma \in\{ \pm\}} \sigma \cos \left(C_{-} t\right) B_{-} X(m, m, m+1, \sigma)+\sigma \sin \left(C_{-} t\right)\left[\sigma \frac{2 \pi m}{d}+\dot{\xi}\left(2 v_{s}^{*} t\right) B_{+}\right] X(m, m, m, \sigma)+\mathrm{Ex}
\end{aligned}
$$

where

$$
\begin{aligned}
X\left(n_{\omega}, n_{1}, n_{2}, \sigma\right) \approx & -\frac{1}{A d^{2}} \frac{8\left(2-\delta_{n_{\omega}, 0}\right)}{B_{-}^{2}-\left[\dot{\xi}\left(2 v_{s}^{*} t\right) B_{+}+\sigma \frac{2 \pi n_{\omega}}{d}\right]^{2}} \sin \left[A \frac{d}{4}+n_{1} \frac{\pi}{2}\right] \\
& \times \sin \left[-B \frac{d}{4}+B_{+} \xi\left(2 v_{s}^{*} t\right)-C_{+} t \chi\left(2 v_{s}^{*} t\right)-\sigma n_{\omega} \frac{2 \pi v_{F}^{*} t}{d}\left(1-\frac{v_{s}^{*}}{v_{F}^{*}}\right)+n_{2} \frac{\pi}{2}\right] .
\end{aligned}
$$

The term Ex in (24) stands for the foregoing expressions with interchanged $B_{+} \Leftrightarrow B_{-}, C_{+} \Leftrightarrow-C_{-}$, and switched sign of $v_{s}^{*}$. Furthermore, we used the abbreviation $A \equiv A_{+}+A_{-}, B \equiv B_{+}+B_{-}$.

We are now able to calculate the conductivity contribution $\tilde{\sigma}_{i i}^{n}$ of the $n$th energy valley by using (17), (21), and (24), leading to

$$
\begin{aligned}
\tilde{\sigma}_{x x}^{n} \approx & \frac{e^{2}}{h} \frac{\pi}{2} \hat{\alpha}_{0}^{2} \frac{1}{\Gamma_{n}}, \quad \tilde{\sigma}_{y y}^{n} \approx \frac{e^{2}}{h} \frac{\pi}{2} \frac{1}{\hat{\alpha}_{0}^{2}} \frac{1}{\Gamma_{n}} \sum_{\sigma \in\{L, R\}} \operatorname{Re}\left[\mathcal{C}_{0}+\mathcal{C}_{1}\right]\left\{\operatorname{Re}\left[\mathcal{C}_{0}\right]+\operatorname{Re}\left[\mathcal{C}_{1}\right]\left[\frac{1}{\pi}\left\{\vartheta_{\sigma}^{n}-\frac{1}{2} \sin \left(2 \vartheta_{\sigma}^{n}\right)\right\}-I_{2}\left(\frac{\Gamma_{n} \Delta k_{y}^{n, \sigma} d}{\pi}, \vartheta_{\sigma}^{n}\right)\right]\right. \\
& \left.-\operatorname{Im}\left[\mathcal{C}_{1}\right]\left[I_{1}\left(\frac{\Gamma_{n} \Delta k_{y}^{n, \sigma} d}{\pi}\right)+I_{3}\left(\frac{\Gamma_{n} \Delta k_{y}^{n, \sigma} d}{\pi}\right)\right]\right\} .
\end{aligned}
$$


Terms containing $\mathcal{C}_{m}$ with $m \geqslant 2$ are neglected here which can be justified numerically. The angle $\vartheta_{\sigma}^{n}$ is given by tan $\left(\vartheta_{\sigma}^{n}\right)=$ $\left(\Gamma_{n} d \Delta k_{y}^{n, \sigma}\right) /\left[\pi^{2}-\left(\Gamma_{n} d \Delta k_{y}^{n, \sigma}\right)^{2}\right]^{1 / 2}$. The functions $I_{1}, \ldots, I_{3}$ are calculated from $(21)$ for $t \rightarrow \infty$ as

$$
\begin{aligned}
I_{1}(x) & =\frac{2}{\pi^{2}} x+\frac{2}{\pi^{2}} \operatorname{sgn}\left[1-x^{2}\right] \operatorname{Re}\left\{\frac{a(x)}{4}\left[\csc ^{2}\left(\frac{a(x)}{2}\right)-\sec ^{2}\left(\frac{a(x)}{2}\right)\right]-\sum_{\sigma \in\{ \pm\}} \sigma\left[a(x) \log \left(1+\sigma e^{i a(x)}\right)+i \operatorname{Li} 2\left(\sigma e^{i a(x)}\right)\right]\right\}, \\
I_{2}(x, \varphi) & =\frac{2}{\pi x^{2}} \sin ^{3}(\varphi)|\cos (\varphi)|, \quad I_{3}(x)=\frac{4}{\pi^{2}} x\left(\sqrt{x^{2}-1} a(x)-1\right),
\end{aligned}
$$

where $a(x) \equiv \arctan \left(1 / \sqrt{x^{2}-1}\right)$ and $\mathrm{Li}_{2}$ is the dilogarithm function.

Here the term $I_{1}$ is calculated from the first summand in the integral on the right-hand side of (21). For the calculation of $I_{2}, I_{3}$ we used the last term in (21). Furthermore we took into account in Eq. (26) the degeneracy of the $\mathbf{K}$ and $\mathbf{K}^{\prime}$ valleys and the spin degeneracy.

We obtain from (26) that the conductivity $\tilde{\sigma}_{x x}^{n}$ does not depend on $v_{s}$, whereas $\tilde{\sigma}_{y y}^{n}$ shows a strong $v_{s}$ dependence. Equation (23) shows that for $v_{s}=0$ the only finite term in $\tilde{\sigma}_{y y}^{n}$ is the term proportional to $\operatorname{Re}\left[\mathcal{C}_{0}\right]^{2}$ in (26). In order to derive this term we made use of the following integral $\lim _{t \rightarrow \infty} \int_{0}^{\infty} d k \sin (2 k t) / k=\pi / 2$. With the help of $\operatorname{Re}\left[\mathcal{C}_{0}\right]=$ $|\Gamma|$ for $v_{s}=0, \tilde{\sigma}_{y y}^{n}$ is reduced to $\tilde{\sigma}_{y y}^{n}=\delta_{n, 0} \Gamma_{n} e^{2} \pi / 2 h$. Furthermore we find for $\tilde{\sigma}_{x x}^{0}$ a divergence at SL potentials where $\tilde{V} \in \mathbb{N} \pi$ for general velocities. The same thing holds for $\tilde{\sigma}_{y y}^{0}$ but here we must demand $v_{s}>0$ where now $\operatorname{Re}\left[\mathcal{C}_{0}\right] \neq|\Gamma|$, i.e., $\operatorname{Re}\left[\mathcal{C}_{0}\right] \neq 0$ in general. The origin of these divergences comes from the vanishing of $\Gamma_{0}$ in the denominator in the right-hand side of (26). This term is already existent in (21). The reason for this vanishing is based on the flatness of the energy band (1) at the central Dirac point in the $k_{y}$ direction at SL potentials where $\tilde{V} \in \mathbb{N} \pi$. In the next section [cf. Eq. (39)] we show for the $v_{s}=0$ conductivity, by going beyond the inner-valley approximation used here, that $\tilde{\sigma}_{y y}$ is exactly vanishing only for $\tilde{V}=\pi$. All this leads us to the following remarkable fact: An infinite large SL graphene sample is an ideal motion detector at SL potentials where the first new Dirac point emerges, i.e., at $\tilde{V}=\pi$. There $\tilde{\sigma}_{y y}$ is vanishing for $v_{s}=0$ and jumps to infinity for $v_{s} \neq 0$.

From (19) and (21), the divergence of $\tilde{\sigma}_{y y}^{0}$ at $\tilde{V} \in \mathbb{N} \pi$ has its origin in the approximation that we used an infinite ballistic time $t \sim t_{b}$ in calculating the response. This is not really valid for a finite system where $t_{b} \sim L / v_{f}$ and $L$ is the length of the sample. By repeating the discussion below (18) but now using the energy (1), $\epsilon_{s} \approx s \hbar v_{F}\left(k_{x}^{2}+d^{4} k_{y}^{6} / 64 \tilde{V}^{4}\right)^{1 / 2}$ at small momenta for $\tilde{V} \in \mathbb{N} \pi$ leads to $\tilde{\sigma}_{i i}^{0}$ in (26) with a finite cutoff at $1 / \Gamma_{0} \sim\left(\tilde{V} t_{b}\right)^{2 / 3}$. In the following we calculate from (19) the conductivities $\sigma_{i i}^{0}$ at $\tilde{V} \in \mathbb{N} \pi$ in leading order in $1 / t_{b}$ for $t_{b} \rightarrow \infty$. The results are $\tilde{\sigma}_{i i} \approx \tilde{\sigma}_{i i}^{0}$ with

$$
\begin{aligned}
\tilde{\sigma}_{x x}^{0}= & \frac{e^{2}}{h} \frac{\sqrt{3}}{4^{2 / 3} \sqrt{\pi}} \frac{\Gamma(1 / 6) \Gamma(1 / 3)}{\Gamma(2 / 3)}\left(\tilde{V} \frac{v_{F} t_{b}}{d}\right)^{2 / 3}, \\
\tilde{\sigma}_{y y}^{0}= & \frac{e^{2}}{h} \frac{\sqrt{3}}{4^{2 / 3} \sqrt{\pi}} \frac{\Gamma(1 / 6) \Gamma(1 / 3)}{\Gamma(2 / 3)}\left(\tilde{V} \frac{v_{F} t_{b}}{d}\right)^{2 / 3} \\
& \times \operatorname{Re}\left[\mathcal{C}_{0}+\mathcal{C}_{1}\right] \operatorname{Re}\left[\mathcal{C}_{00}\right],
\end{aligned}
$$

where $\mathcal{C}_{00}=\left(v_{s} / d\right) \int_{0}^{d / v_{s}} d t \mathcal{C}_{0}$. By using (24) we obtain

$$
\operatorname{Re}\left[\mathcal{C}_{00}\right]=\frac{32 \sin \left(A \frac{d}{4}\right)}{B_{+} B_{-} A d^{3}}\left[\cos \left(\left\{B_{+}-B_{-}\right\} \frac{d}{4}\right)-\cos \left(B \frac{d}{4}\right)\right] .
$$

In Fig. 2 we plot $\tilde{\sigma}_{y y}$ for $v_{s} / v_{F}=0.1$ (left panel) and $v_{s} / v_{F}=0.01$ (right panel), as well as for $v_{s}=0$ (horizontal curves) at various $\tilde{V}$ values. The most interesting $\tilde{V}$ values are where for a certain $v_{s}$ the signal $\tilde{\sigma}_{y y}$ is largest. In particular, the signal-to-background ratio, i.e., $\tilde{\sigma}_{y y}$ divided by $\tilde{\sigma}_{y y}$ for $v_{s}=0$, should be large. We obtain from the figure and (26) as well as (28) that for a finite system and $v_{s} \ll v_{F}$, the SL potential region where $\tilde{V} \sim v_{F} / v_{s}$ and $\tilde{V} \in \mathbb{N} \pi$ gives the best results. We plot this in Fig. 2 for $\tilde{V}=\left[0.3 v_{F} / v_{s}\right] \pi$. This is chosen so that the curves do not show a higher-order $v_{s}$-Fourier behavior according to (24). We note that, in principle, a graphene velocity detector based on a SL considered here could also attain a large signal-to-background conductivity for small velocity differences by using large SL potentials $\tilde{V} \sim v_{F} / \Delta v_{s}$. This is due to the phase factors in (24).

In addition to the oscillation frequencies $\sim 2 \pi v_{F}^{*} / d$ and $\sim 2 \pi v_{s}^{*} / d$ we also find from (24) and Fig. 2 a much smaller oscillation frequency $\sim C_{ \pm}$for the conductivity contribution of the side valleys becoming relevant only on very large time scales. One can show that due to its nonzero velocity, the SL transfers additional energy and momentum to an electron passing its potential steps such that the electron velocity oscillates between $\pm v_{F}\left(1-\tilde{k}_{y}^{2} / 2\right)$ and $\pm v_{F}[1-$ $\left.\tilde{k}_{y}^{2}\left(v_{F} \pm v_{s}\right)^{2} / 2\left(v_{F} \mp v_{s}\right)^{2}\right]$. Due to this velocity difference the electron picks up an additional oscillating phase proportional to $t$ represented by the last term in (15). This leads to the long wave-conductivity oscillations shown in Fig. 2.

To complete our discussion, we finally calculate the quasiparticle velocities in the $x$ and $y$ directions for electrons in the $\mathbf{u}_{ \pm 1}^{v_{s}}$ state where now $E_{\mathrm{dc}}=0$. The knowledge of these velocities is useful in quantum pumping experiments. ${ }^{24,25} \mathrm{We}$ obtain from (1) and (8)

$$
\begin{aligned}
v_{x} & =v_{F}\left\langle\mathbf{u}_{ \pm 1}^{v_{s}}\left|\sigma_{x x}\right| \mathbf{u}_{ \pm 1}^{v_{s}}\right\rangle=\frac{\partial \epsilon_{ \pm}}{\hbar \partial k_{x}}, \quad v_{y}=v_{F}\left\langle\mathbf{u}_{ \pm 1}^{v_{s}}\left|\sigma_{y y}\right| \mathbf{u}_{ \pm 1}^{v_{s}} \mid\right\rangle \\
& =\frac{\partial \epsilon_{ \pm}}{\hbar \partial k_{y}}|\mathcal{P}| \approx \frac{\partial \epsilon_{ \pm}}{\hbar \partial k_{y}} \operatorname{Re}\left[\mathcal{C}_{0}+\mathcal{C}_{1}\right] .
\end{aligned}
$$

This means that similar to the above conductivity considerations we obtain no time dependence of $v_{x}$, in contrast to $v_{y}$. As in the nonmoving system ${ }^{26}$ there is a collimation of the electron motion in the $x$ direction, i.e., $\left|v_{y}\right| \ll\left|v_{x}\right|$ for potentials were 


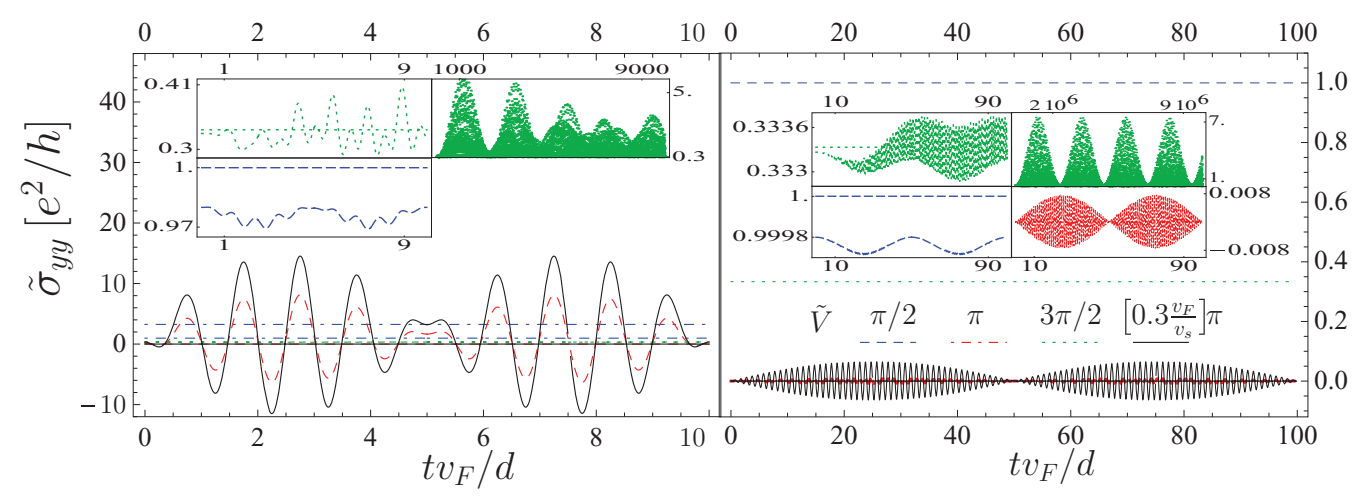

FIG. 2. (Color online) We show the conductivity $\tilde{\sigma}_{y y}$ orthogonal to the SL calculated for velocities $v_{s} / v_{F}=0.1$ (left panel) and $v_{s} / v_{F}=0.01$ (right panel) as a function of the dimensionless time. We plot the curves for various SL potentials $\tilde{V}$ by using (26) for $\tilde{V} \notin \mathbb{N} \pi,(28)$ for $\tilde{V} \in \mathbb{N} \pi$, and ballistic times $\left(v_{F} t_{b} / d\right)^{2 / 3}=1000$. The horizontal curves show $\tilde{\sigma}_{y y}$ for $v_{s}=0$. Insets in both panels show a zoom in of the corresponding curves in the main panels (upper left: $\tilde{V}=3 \pi / 2$; lower left: $\tilde{V}=\pi / 2$; lower right: $\tilde{V}=\pi$ ). We also add for $\tilde{V}=3 \pi / 2$ in the upper right insets $\tilde{\sigma}_{y y}$ for larger times.

$\tilde{V} \approx \mathbb{N} \pi$ and momenta $k_{y}$ near the central Dirac point. Here we use $|\mathcal{P}| \leqslant 1$.

\section{OUTER-VALLEY TRANSPORT CONTRIBUTIONS}

Next, we discuss the conductivity contributions of the outerenergy valleys where $\hat{\alpha}_{0} \ll 1$. We obtain from (1) that the new Dirac points are located at $k_{y}^{n} d=2\left[\tilde{V}^{2}-(\pi n)^{2}\right]^{1 / 2}$ where the linearized energy spectrum around these points is given by $\epsilon_{s}=s \hbar v_{F}\left[\hat{\alpha}_{0}^{4} k_{x}^{2}+\Gamma_{n}^{2}\left(k_{y}-k_{y}^{n}\right)^{2}\right]^{1 / 2}$. The effective $y$-velocity coefficient is now given by $\Gamma_{n}=\left[\tilde{V}^{2}-(\pi n)^{2}\right] / \tilde{V}^{2}$ and $\hat{\alpha}_{0}=$ $\pi n / \tilde{V}$. This means that the outer-valley regime $\hat{\alpha}_{0} \ll 1$ is fulfilled for those valleys where $\pi n / \tilde{V} \ll 1$.

We obtain now from Sec. II for the space evolution operator (5) of the nonmoving system

$$
\lambda_{x_{0}}(x) \approx \frac{1}{\alpha_{\epsilon_{s}}(x)} \mathbf{M} \sin \left[2 \alpha_{\epsilon_{s}}(x) \frac{\left(x-x_{0}\right)}{d}\right]
$$

to leading order in $\hat{\alpha}_{0}$. The corresponding lowest-band eigenfunctions $\mathbf{u}_{s}^{0}$ can be interpreted by electrons which are fully backscattered close to the potential steps for $\left|\epsilon_{s}\right| d / \hbar v_{F} \ll \hat{\alpha}_{0}$. This is just the opposite situation of the inner-valley transport contributions discussed in Sec. III where we got a complete transmission through the potential steps. This interpretation is even justified by discussing the scattering of electrons on a single potential step in the momentum regime $\hat{\alpha}_{0} \ll 1$. In this regime $\mathbf{u}_{s}^{v_{s}}$ can now be written as in (13) with the substitution of the spinor part $\left(s k_{x} /\left|k_{x}\right|, 1\right) \rightarrow\left(-i s k_{y} /\left|k_{y}\right|, 1\right)$. For the moving lattice we concentrate, in the following, on a particle moving in a potential $\pm V$ in the region $-v_{s} t \leqslant x \leqslant d / 2-v_{s} t$.

We now determine a complete set of functions $v_{ \pm}^{j}(x, t)$ fulfilling the quasirelativistic Klein-Gordon equation with a potential $V(x)= \pm V$ in the region $-v_{s} t \leqslant x \leqslant-v_{s} t+$ $d^{*} / 2$. They further satisfy the zero-boundary conditions $v_{ \pm}^{j}\left(-v_{s} t, t\right)=v_{ \pm}^{j}\left(-v_{s} t+d^{*} / 2, t\right)=0$. These properties unambiguously define the functions $v_{ \pm}^{j}(x, t)$. The distance $d^{*}$ has a small modification to the distance $d$ for $\left|\epsilon_{s}\right| d / \hbar v_{F} \ll \hat{\alpha}_{0}$ determined by $\alpha_{\epsilon_{s}} d^{*} / d=\pi n$ for the $n$th energy valley, i.e., $\alpha_{0}=$ $\pi n$. The wave functions $v_{ \pm}^{j}(x, t)$ consist of a superposition of two Klein-Gordon wave-function solutions. The momenta of both Klein-Gordon wave functions can be formally derived from the zero-boundary conditions. More concretely, the two corresponding momenta are given by a particle initial momentum and its reflected momentum at the boundary. In the quasinon-relativistic limit valid for $v_{F}\left|k_{x}^{j} / k_{y}\right|,\left|v_{s}\right| \ll v_{F}$ we obtain for these momenta $k_{x}^{j} \pm v_{s}\left|k_{y}\right| / v_{F}$ and $-k_{x}^{j} \pm v_{s}\left|k_{y}\right| / v_{F}$ with $j \in \mathbb{N}$ and $k_{x}^{j}=2 \pi j / d^{*}$ in the potential $V(x)= \pm V$. The restriction on the quasi-non-relativistic limit is justified for the outer-valley transport contributions in the case $v_{s} \ll v_{F}$. This leads to

$$
\begin{aligned}
v_{ \pm}^{j}(x, t)= & \frac{2}{\sqrt{d^{*}}} e^{ \pm i\left(\hbar v_{F} \sqrt{k_{x}^{j}+k_{y}^{2}}-V\right) t / \hbar} e^{\mp i(1 / 2)\left(v_{s}^{2} / v_{F}\right)\left|k_{y}\right| t} \\
& \times e^{ \pm i\left(v_{s}\left|k_{y}\right| / v_{F}\right)\left(x+v_{s} t\right)} \sin \left[k_{x}^{j}\left(x+v_{s} t\right)\right] .
\end{aligned}
$$

By using (31) with (3)-(7), the wave function $\mathbf{u}_{s}^{v_{s}}$ is then given by

$$
\begin{aligned}
\mathbf{u}_{s}^{v_{s}}(x, t)= & -i \frac{d}{2 \alpha_{0}}\left\{\operatorname{sg}\left[k_{y} V\left(x+v_{s} t\right)\right] \frac{\cos \left(\alpha_{0}\right) \sin \left(\alpha_{0}\right)}{\alpha_{0}} k_{y}^{2} d\right. \\
& \left.+2 \operatorname{sg}\left[k_{y}\right] \frac{\epsilon_{s} \tilde{V}}{\hbar v_{F} \hat{\alpha}_{0}^{2}}+i \operatorname{sg}\left[V\left(x+v_{s} t\right)\right]\left|k_{y}\right| k_{x} d\right\} \\
& \times\left(\begin{array}{c}
\operatorname{sg}\left[k_{y} V\left(x+v_{s} t\right)\right] i \\
1
\end{array}\right) \sum_{j} c_{j}^{\operatorname{sg}\left[V\left(x+v_{s} t\right)\right]} \\
& \times v_{\operatorname{sg}\left[V\left(x+v_{s} t\right)\right]}^{j}(x, t)
\end{aligned}
$$

with

$$
c_{j}^{\operatorname{sg}\left[V\left(x+v_{s} t\right)\right]}=\int_{0}^{d^{*} / 2} d x\left(v_{\mathrm{sg}\left[V\left(x+v_{s} t\right)\right]}^{j}\right)^{*}(x, 0) \sin \left(\alpha_{\epsilon_{s}} \frac{2 x}{d}\right) .
$$

With this wave function in hand we are now prepared to calculate the conductivities $\tilde{\sigma}_{i i}^{n}$ for the outer valleys $\pi n / \tilde{V} \ll$ 1. By using (17) with (19) and (33) we obtain for the 


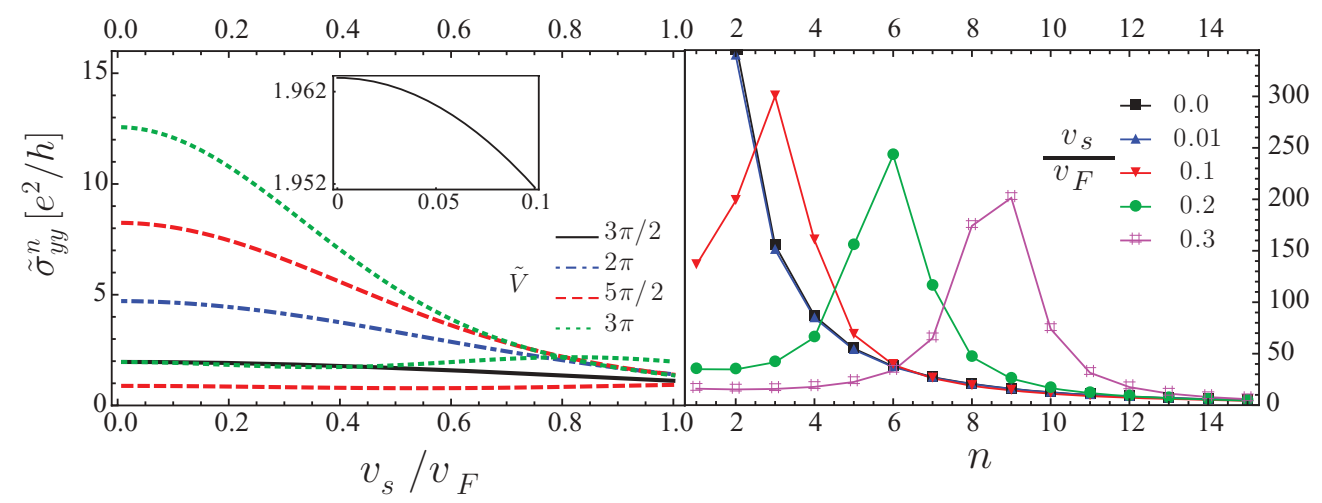

FIG. 3. (Color online) Left panel: Outer valley conductivities $\tilde{\sigma}_{y y}^{n}$ (35) of the $n$th electron side valley as a function of $v_{s} / v_{F}$ for certain SL potentials. For $\tilde{V}=5 \pi / 2$ and $\tilde{V}=3 \pi$, which both consist of two side valleys, the upper curve corresponds to the valley index $n=1$ and the lower curve to $n=2$. Inset shows a zoom in of $\tilde{\sigma}_{y y}^{1}$ for $\tilde{V}=3 \pi / 2$. Right panel: $\tilde{\sigma}_{y y}^{n}$ for $\tilde{V}=30 \pi$ as a function of the valley index $n$ for certain SL velocities $v_{s} / v_{F}$.

conductivities,

$$
\tilde{\sigma}_{x x}^{n} \approx 0, \quad \tilde{\sigma}_{y y}^{n} \approx \frac{e^{2}}{h} \frac{\pi}{2} \frac{1}{\hat{\alpha}_{0}^{2}} \Gamma_{n} Y\left(v_{s}\right)
$$

with $Y\left(v_{s}\right)$ given by

$$
Y\left(v_{s}\right)=\sum_{i, j>0} \frac{n^{2}}{i^{2}}\left|c_{i}\right|^{2}\left|c_{j}\right|^{2}\left(\delta_{i, j}+2 \delta_{i>j}\right) .
$$

Here we use $\left|c_{i}\right|=\left|c_{i}^{ \pm}\right|$and $c_{i}, c_{j}$ in (36) and (34) are calculated with $d^{*} \rightarrow d$.

We find from (35) that the transport contributions of the outer valleys corresponding to $\hat{\alpha}_{0} \ll 1$ show no time fluctuations. This is not based on the quasi-non-relativistic approximation used above. We show in Fig. $3 \tilde{\sigma}_{y y}^{n}$ for the outer valleys and various SL potentials $\tilde{V}$ and velocity fractions $v_{s} / v_{F}$. Most pronounced, the curves in the right panel show a conductivity peak at valley indices where $n \approx n_{0}$. Here $n_{0}$ is given by $n_{0}=\left[\left|v_{s} / v_{F}\right|\left(\tilde{V}^{2}-(\pi n)^{2}\right)^{1 / 2} / \pi\right]$. This conductivity peak is also observed from (35) and (36) by taking into account that in a rough approximation we have $\left|c_{i}\right|^{2} \approx\left(\delta_{i, n+n_{0}}+\delta_{i,\left|n-n_{0}\right|}\right) / 2$ leading to

$$
Y\left(v_{s}\right) \approx \frac{1}{4} \frac{n^{2}}{\left|n-n_{0}\right|^{2}}+\frac{3}{4} \frac{n^{2}}{\left|n+n_{0}\right|^{2}} .
$$

All this means that for $\tilde{V} \gtrsim \pi v_{F} / v_{s}$ with $\tilde{V} \gg 1$ we obtain a large conductivity signal where the conductivity modification due to the motion of the SL is of similar magnitude as the conductivity value of the nonmoving SL. Something similar applies for the detection of small velocity differences $\Delta v_{s}$ where now we have $\tilde{V} \gtrsim \pi v_{F} / \Delta v_{s}$ in order to obtain a large signal-to-background value. By comparing the conductivity values $\tilde{\sigma}_{y y}^{n}$ for the inner valleys (26) (Fig. 2) and the outer valleys (35) (Fig. 3) we find, at least for $\tilde{V} \gg 1$ and $\tilde{V} \not \mathbb{N} \pi$, that the outer-valley contributions are dominant.

Next, we calculate the effective particle velocities for electrons in the outer valleys defined in (30), where now again $E_{\mathrm{dc}}=0$. By using (32) and (33) we obtain

$$
v_{x}=\frac{\partial \epsilon_{ \pm}}{\hbar \partial k_{x}}, \quad v_{y}=\frac{\partial \epsilon_{ \pm}}{\hbar \partial k_{y}} .
$$

This shows that there is no $v_{s}$-correction term in contrast to the inner-valley case (30) for $v_{y}$. This is caused by the fact that in the outer-valley regime electrons are approximately fully reflected, and thus the total probability of finding an electron between $-v_{s} t$ and $-v_{s} t+d / 2$ is conserved.

The nontrivial dependence of the conductivities on the SL velocity forced us to treat the conductivity contributions for the inner and outer valleys separately. This separation is no longer necessary when calculating the conductivities for the nonmoving SL. For this we use the full oscillatory wave function (3)-(7) with (17) and (21). This leads us to the following $v_{s}=0$ conductivities

$$
\tilde{\sigma}_{x x}^{n}=\frac{e^{2}}{h} \frac{\pi}{2} \hat{\alpha}_{0}^{2} \frac{1}{\Gamma_{n}}, \quad \tilde{\sigma}_{y y}^{n}=\frac{e^{2}}{h} \frac{\pi}{2} \frac{1}{\hat{\alpha}_{0}^{2}} \Gamma_{n} .
$$

Note here that the magnitudes of $\hat{\alpha}_{0}$ and $\Gamma_{n}$ correspond to the outer-valley values discussed above Eq. (32) for $n \neq 0$ and to the $n=0$ values discussed above Eq. (12). Similar expressions as in (39) were calculated before within the dc vector potential gauge $\mathbf{A}=0$, leading as in pristine graphene to a small overall numerical prefactor correction to our result (39). ${ }^{18}$ The disadvantage of the calculation in Ref. 18 lies in the strong dependence of this prefactor on the order of taking the zero-temperature, zero-frequency, and zero-damping limit. This does not happen in our calculation. ${ }^{8}$

\section{SUMMARY}

Summarizing, we have considered the dc transport in neutral graphene undulated by a unidirectional moving superlattice potential with $v_{s} \lesssim v_{F}, V d / \hbar$. While the response along the direction of the SL wave vector is vanishing, the dependence is dramatic in the orthogonal direction. In particular, we find for potentials where the first new Dirac point emerges, i.e., at $\tilde{V}=\pi$, that the infinite large graphene sample is a perfect motion detector. The orthogonal dc conductivity is vanishing for zero velocity and jumps to infinity at nonzero SL velocity. A large conductivity signal with a high signal-to-background ratio is reached for the finite but large graphene system when $\tilde{V} \in \mathbb{N} \pi$. The time fluctuating contribution to the conductivity is largest when $\tilde{V} \sim v_{F} / v_{s}$. 
All this was derived from the inner-valley contributions to the conductivities.

Next we have calculated the conductivity contributions of the outer valleys. The conductivity contributions parallel to the SL wave vector are vanishing. In the orthogonal direction they are large, time-independent, and exhibit a peak as a function of the valley index. For $\tilde{V} \gg 1$ and $\tilde{V} \gtrsim \pi v_{F} / v_{s}$ the conductivity modifications due to a moving SL are of similar magnitude as the conductivity values of the stagnant SL. Note that for $\tilde{V} \gg 1$ the outer-valley conductivity contributions are dominant over the inner-valley contributions, at least for $\tilde{V} \not \mathbb{N} \pi$. Finally, we have calculated the conductivities of the nonmoving SL without the need of a separate calculation for the inner and outer valleys. Due to its intrinsic low-noise level, ${ }^{9}$ our results could be useful for graphene as a nanophysical motion detector device, or even for general sensors based on the surface acoustic wave technology. ${ }^{27}$

\section{APPENDIX A: SOLVING THE HAMILTON-JACOBI EQUATION (12)}

Here we outline the calculation of (15) by solving the Hamilton-Jacobi Equation (12) to first order in $\tilde{k}_{y}^{2}$. This is done with the help of a generalized characteristic method. ${ }^{23}$ The solution is based on the one-particle quasirelativistic orbit $x(t)$ in a moving potential $V\left(x+v_{s} t\right)$. With the help of this solution, $S_{ \pm}^{v_{s}}(x, t)$ is given by the action integral

$$
\begin{aligned}
S_{ \pm}^{v_{s}}(x, t)= & \int_{0}^{t} d t^{\prime}\left\{\frac{v_{F} \hbar^{2} k_{y}^{2}}{\sqrt{p^{2}\left(t^{\prime}\right)+\hbar^{2} k_{y}^{2}}} \operatorname{sg}\left\{V\left[x\left(t^{\prime}\right)+v_{s} t^{\prime}\right]\right\}\right. \\
& \left.-V\left[x\left(t^{\prime}\right)+v_{s} t^{\prime}\right]\right\}+S_{ \pm}^{0}\left(x_{0}, 0\right) .
\end{aligned}
$$

Here $x\left(t^{\prime}\right)$ is the particle trajectory with $x(0)=x_{0}, x(t)=x$. The particle momentum is given by

$$
p\left(t^{\prime}\right)=\partial_{x} S_{ \pm}^{v_{s}}\left(x\left(t^{\prime}\right), t^{\prime}\right)
$$

and the quasirelativistic velocity by

$$
\dot{x}\left(t^{\prime}\right)=-v_{F} \operatorname{sg}\left\{V\left[x\left(t^{\prime}\right)+v_{s} t^{\prime}\right]\right\} \frac{p\left(t^{\prime}\right)}{\sqrt{p^{2}\left(t^{\prime}\right)+\hbar^{2} k_{y}^{2}}} .
$$

We note now that it is much easier to determine $x\left(t^{\prime}\right)$ by solving the set of equations above for small $\tilde{k}_{y}$, instead of solving the second-order quasirelativistic Newton equation. From this we obtain (15).

\section{APPENDIX B: A GUIDELINE TO REPRODUCE THE FORMULAS}

Here we give a short guideline for readers who would like to reproduce the formulas in this paper.

\section{Equations (14)-(16)}

We first solve (A1)-(A3) in leading order in $\tilde{k}_{y}^{2}$, i.e., for $k_{y}=0$. This leads with (A3) to the particle velocities up to the next leading order in $\tilde{k}_{y}^{2}$. We obtain

$\dot{x}(t)=v_{0} \delta_{\mathrm{sg}\left[V\left(x_{0}\right)\right], \operatorname{sg}\left\{V\left[x(t)+v_{s} t\right]\right\}}+v_{1}\left(1-\delta_{\mathrm{sg}\left[V\left(x_{0}\right)\right], \operatorname{sg}\left\{V\left[x(t)+v_{s} t\right]\right\}}\right)$

with $\quad v_{0}= \pm v_{F}\left(1-\tilde{k}_{y}^{2} / 2\right) \quad$ and $\quad v_{1}= \pm v_{F}\left[1-\tilde{k}_{y}^{2}\left(v_{F} \pm\right.\right.$ $\left.\left.v_{s}\right)^{2} / 2\left(v_{F} \mp v_{s}\right)^{2}\right]$. With these velocities in hand one can derive the particle's action $S_{ \pm}^{v_{s}}$ to order $\tilde{k}_{y}^{2}$ by using (A1) and (A3). Here we have used the identity $x_{0}=x-\left(v_{0}+v_{1}\right) t / 2+\Delta x_{0}$ where

$$
\begin{aligned}
\Delta x_{0} \approx & -\operatorname{sg}\left[V\left(x_{0}\right)\right] \frac{v_{F}^{2} v_{s}}{v_{s} \mp v_{F}} \frac{1}{v_{s}^{2}-v_{F}^{2}} \\
& \times\left\{\xi\left(x+v_{s} t\right)-\xi\left[x-\left(v_{0}+v_{1}\right) t / 2\right]\right\}
\end{aligned}
$$

during the derivation. Equation (B2) is valid in the next-toleading order in $\tilde{k}_{y}^{2}$. It connects the starting point $x_{0}$ of the trajectory with its end point $x$.

We calculated $v_{0}, v_{1}$ in (B1) by using the approximation $\left|\epsilon_{s}\right| \ll V$. Going beyond this approximation could lead for $S_{ \pm}^{v_{s}, t}(15)$ to small possible additional terms of the order $\pm t \epsilon_{s} \tilde{k}_{y}^{2}\left(v_{s} / v_{F}\right)$. Such terms would then result in a small time-independent numerical prefactor correction in the oscillatory side-valley conductivity $\tilde{\sigma}_{y y}^{n}$ for $n>0$ of the order $\left(k_{y}^{n} d / \tilde{V}\right)^{2}\left(v_{s} / v_{F}\right)^{2}(26)$. The conductivities $\tilde{\sigma}_{x x}^{n}$ would get a similar small prefactor correction. Finally we note, that by setting $Z_{ \pm}^{1}, Z_{ \pm}^{2}=0$ in (14)-(16), the corresponding action $S_{ \pm}^{v_{s}}$ is given by (A1) where now the particle trajectory and the particle momentum is calculated from the uniform velocity $v_{1}=v_{0}= \pm v_{F}\left(1-\tilde{k}_{y}^{2} / 2\right)$.

\section{Equations (23)-(25)}

In order to derive (24) and (25) from (23) we used $|A| \gtrsim|B|$ for $v_{s} \lesssim v_{F}$. Then we obtain for not too large ballistic times $C_{ \pm} t_{b} \ll 1$ but also for large times $C_{ \pm} t_{b} \gtrsim 1$ where now we have to restrict ourselves to the most relevant low-frequency Fourier components $\mathcal{C}_{m}$ with $m \lesssim 1$, such that

$$
\begin{aligned}
|P|\left(t^{\prime}, t\right) \approx & \frac{4}{A d} \sin \left[(A+B) \frac{d}{4}-B_{+} \xi\left(-v_{F}^{*} t^{\prime}-v_{s}^{*} t\right)\right. \\
& -B_{-} \xi\left(v_{F}^{*} t^{\prime}-v_{s}^{*} t\right)-C_{+} t \chi\left(-v_{F}^{*} t^{\prime}-v_{s}^{*} t\right) \\
& \left.-C_{-} t \chi\left(v_{F}^{*} t^{\prime}-v_{s}^{*} t\right)\right],
\end{aligned}
$$

where $|P|(t)=|P|(t, t)$. In order to calculate $\mathcal{C}_{m} \quad(23)$ we can use for $v_{s} \lesssim v_{F}$ the approximation $C_{m} \approx$ $\left(d / v_{F}^{*}\right) \int_{0}^{v_{F}^{*} / d} d t^{\prime}|P|\left(t^{\prime}, t\right) e^{-i 2 \pi m v_{F}^{*} t^{\prime} / d}$, which then leads to the expressions (24) and (25).

\section{Equations (26) and (27)}

The integrals $I_{1}(x)$ and $I_{3}(x)$, which are the terms proportional to $\operatorname{Im}\left[\mathcal{C}_{1}\right]$ at the right bottom of Eq. (26), are calculated 
by using (21) and (22),

$$
\begin{aligned}
I_{1}(x)= & \frac{1}{2 \pi^{2}} \int_{-x}^{+x} d k_{y} \int_{-\infty}^{+\infty} d k_{x} \frac{k_{x}^{2}}{{\sqrt{k_{x}^{2}+k_{y}^{2}}}^{3}} \\
& \times \sum_{\sigma \in\{ \pm\}} \frac{\sigma}{\sqrt{k_{x}^{2}+k_{y}^{2}}-\sigma} \\
I_{3}(x)= & \frac{1}{2 \pi^{2}} \int_{0}^{2 \pi} d \vartheta \sum_{\sigma \in\{ \pm\}} \sigma \frac{\sin ^{2}(\vartheta) \sqrt{1+\tan ^{2}(\vartheta)}}{\sqrt{1+\tan ^{2}(\vartheta)}-\sigma / x} .
\end{aligned}
$$

${ }^{1}$ S. V. Morozov, K. S. Novoselov, M. I. Katsnelson, F. Schedin, D. C. Elias, J. A. Jaszczak, and A. K. Geim, Phys. Rev. Lett. 100, 016602 (2008).

${ }^{2}$ X. Du, I. Skachko, A. Barker, and E. Y. Andrei, Nat. Nanotechnol. 3, 491 (2008).

${ }^{3}$ K. I. Bolotin, K. J. Sikes, J. Hone, H. L. Stormer, and P. Kim, Phys. Rev. Lett. 101, 096802 (2008).

${ }^{4}$ H. A. Castro Neto, F. Guinea, N. M. R. Peres, K. S. Novoselov, and A. K. Geim, Rev. Mod. Phys. 81, 109 (2009).

${ }^{5}$ M. Lewkowicz, B. Rosenstein, and D. Nghiem, Phys. Rev. B 84, 115419 (2011).

${ }^{6}$ M. I. Katsnelson, Eur. Phys. J. B 51, 157 (2006).

${ }^{7}$ J. Tworzydlo, B. Trauzettel, M. Titov, A. Rycerz, and C. W. J. Beenakker, Phys. Rev. Lett. 96, 246802 (2006).

${ }^{8}$ M. Lewkowicz and B. Rosenstein, Phys. Rev. Lett. 102, 106802 (2009); H. C. Kao, M. Lewkowicz, and B. Rosenstein, Phys. Rev. B 82, 035406 (2010).

${ }^{9}$ F. Schedin, A. K. Geim, S. V. Morozov, E. W. Hill, P. Blake, M. I. Katsnelson, and K. S. Novoselov, Nature Mater. 6, 652 (2007).

${ }^{10}$ E. W. Hill, A. Vijayaragahvan, and K. Novoselov, IEEE Sens. J. 11, 3161 (2011).

${ }^{11}$ P. Thalmeier, B. Dóra, and K. Ziegler, Phys. Rev. B 81, 041409(R) (2010).

${ }^{12}$ V. I. Talyanskii, D. S. Novikov, B. D. Simons, and L. S. Levitov, Phys. Rev. Lett. 87, 276802 (2001).

${ }^{13}$ C.-H. Park, L. Yang, Y.-W. Son, M. L. Cohen, and S. G. Louie, Phys. Rev. Lett. 101, 126804 (2008).
The terms proportional to $\operatorname{Re}\left[\mathcal{C}_{1}\right]$ in Eq. (26) were also derived from (21) with (22) by making use of the identity $\lim _{t \rightarrow \infty} \frac{1}{f(k)} \sin [f(k) t]=\pi \delta[f(k)]$ for an arbritary function $f$. Here $\delta(x)$ is the Dirac $\delta$ function.

\section{Equations (34)-(36)}

Here we use (33) with (32) in (19) and (17). With the help of a small $\epsilon_{s}$ expansion of the exponents in (32) we obtain (34)-(36) by using $\lim _{t \rightarrow \infty} \int_{0}^{\infty} d k \sin (2 k t) / k=\pi / 2$. Note that we get a contribution only from the first term in Eq. (19) in this calculation, which leads to the final result (35) for $t \rightarrow \infty$.
${ }^{14}$ M. Yankowitz, J. Xue, D. Cormode, J. D. Sanchez-Yamagishi, K. Watanabe, T. Taniguchi, P. Jarillo-Herrero, P. Jacquod, and B. J. LeRoy, Nat. Phys. 8, 382 (2012).

${ }^{15}$ L. Brey and H. A. Fertig, Phys. Rev. Lett. 103, 046809 (2009).

${ }^{16}$ J. Dietel and H. Kleinert, Phys. Rev. B 84, 121404(R) (2011).

${ }^{17}$ C.-H. Park, Y.-W. Son, L. Yang, M. L. Cohen, and S. G. Louie, Phys. Rev. Lett. 103, 046808 (2009).

${ }^{18}$ P. Burset, A. L. Yeyati, L. Brey, and H. A. Fertig, Phys. Rev. B 83, 195434 (2011).

${ }^{19}$ M. Barbier, P. Vasilopoulos, and F. Peeters, Philos. Trans. R. Soc. London, Ser. A 368, 5499 (2010).

${ }^{20}$ J. Sun, H. A. Fertig, and L. Brey, Phys. Rev. Lett. 105, 156801 (2010).

${ }^{21}$ D. P. Arovas, L. Brey, H. A. Fertig, E.-A. Kim, and K. Ziegler, New J. Phys. 12, 123020 (2010).

${ }^{22}$ M. Barbier, P. Vasilopoulos, and F. M. Peeters, Phys. Rev. B 81, 075438 (2010).

${ }^{23}$ V. Maslov and M. V. Fedoriuk, Semi-Classical Approximation in Quantum Mechanics (Reidel, Dodrecht, Netherlands, 1981).

${ }^{24}$ D. J. Thouless, Phys. Rev. B 27, 6083 (1983).

${ }^{25}$ E. Prada, P. San-Jose, and H. Schomerus, Phys. Rev. B 80, 245414 (2009).

${ }^{26}$ C.-H. Park, Y.-W. Son, L. Yang, M. L. Cohen, and S. G. Louie, Nano Lett. 8, 2920 (2008).

${ }^{27}$ A. V. Mamishev, K. Sundara-Rajan, F. Yang, Y. Du, and M. Zahn, Proc. IEEE 92, 808 (2004). 